\title{
Association between Therapeutic Alliance and Adherence in Outpatient Schizophrenia Patients
}

\author{
Jhin Goo Chang ${ }^{1,4}$, Daeyoung Roh ${ }^{2}$, Chan-Hyung Kim ${ }^{3,4}$ \\ ${ }^{1}$ Department of Psychiatry, Myongi Hospital, Hanyang University College of Medicine, Goyang, ${ }^{2}$ Department of Psychiatry, Hallym University \\ College of Medicine, Chuncheon, ${ }^{3}$ Institute of Behavioral Science in Medicine and ${ }^{4}$ Department of Psychiatry, Yonsei University College of \\ Medicine, Seoul, Korea
}

\begin{abstract}
Objective: Although various clinical factors that affect medication adherence in schizophrenia have been studied, the role of the therapeutic alliance has not been studied in detail. Accordingly, we investigated the association between medication adherence and therapeutic alliance in patients with schizophrenia treated in a community outpatient clinic in Korea.

Methods: In this cross-sectional study, 81 outpatients who met the DSM-IV-TR criteria for schizophrenia were analyzed. Therapeutic alliance was measured via patient-self-report questionnaires consisting of 12 questions, which evaluate both "affective bond" and "collaborative bond" of alliance. We investigated the relationship between medication adherence and therapeutic alliance through correlation and regression analyses.

Results: Overall therapeutic alliance was weakly associated with medication adherence $(r=0.268, p<0.05)$. Among two factors of therapeutic alliance, "affective bond" was associated with adherence $(\mathrm{r}=0.302, p<0.05)$, but collaborative was not. Regression analysis showed that therapeutic alliance significantly predicted medication adherence even after adjustment for duration of treatment, insight, and symptom severity.

Conclusion: Maintaining a favorable therapeutic alliance is associated with medication adherence in schizophrenia. Further, treating patients in a frank and genuine manner might be important to improve adherence.
\end{abstract}

KEY WORDS: Schizophrenia; Physician-patient relations; Medication adherence.

\section{INTRODUCTION}

Patients with schizophrenia must take medicine on a regular basis to control acute positive symptoms and prevent long-term relapse. ${ }^{1,2)}$ However, many such patients do not follow their clinician's recommendations and instead stop taking medications. Among patients whose symptoms are alleviated via medication, 30\% to 50\% do not comply with their clinician's prescriptions after symptoms are relieved, ${ }^{3)}$ and $50 \%$ of inpatients stop taking medicines without discussion with their health care pro-

Received: June 15, 2018 / Revised: October 29, 2018

Accepted: November 15, 2018

Address for correspondence: Chan-Hyung Kim, MD, PhD

Department of Psychiatry, Severance Hospital, Yonsei University College of Medicine, 50 Yonsei-ro, Seodaemun-gu, Seoul 03722, Korea

Tel: +82-2-2228-1620, Fax: +82-2-313-0891

E-mail: spr88@yuhs.ac

ORCID: https://orcid.org/0000-0001-8419-3759 viders within 1 year of discharge. ${ }^{4)}$ Moreover, patients with psychiatric disorders are known to have a higher tendency to avoid taking their medications than are those with physical disorders. ${ }^{5)}$ Non-adherence to medication in schizophrenia remains an unsolved problem that reduces patients' quality of life and incurs a tremendous social cost. ${ }^{6)}$

Various studies have been conducted to identify the determinants of medication non-adherence in patients with schizophrenia. ${ }^{7-11)}$ Impaired illness insight, one of the key features of schizophrenia, has consistently been reported to impact adherence to treatment. ${ }^{7,10,11)}$ Suffering severe psychotic symptoms ${ }^{8)}$ and comorbid substance use disorder $^{12)}$ also showed a significant relationship with nonadherence. The majority of adherence studies have concentrated on the characteristics of patients or disease-related characteristics, since non-adherence in schizophrenia has typically been regarded as an irrational behavior resulting

(ㄷ) This is an Open-Access article distributed under the terms of the Creative Commons Attribution Non-Commercial License (http://creativecommons.org/licenses/by-nc/4.0) which permits unrestricted non-commercial use, distribution, and reproduction in any medium, provided the original work is properly cited. 
from psychotic symptoms or lack of illness insight. Recently, interest in treatment environment factors has grown as it is known that patients' attitudes to treatment have an important impact on adherence. ${ }^{13)}$ Day et al. ${ }^{7}$ reported that modifying patient's negative experiences of treatment could improve adherence through encouraging a positive attitude to treatment.

Therapeutic alliance refers to the collaborative and affective intimacy between a between a therapist and his or her patient, ${ }^{14)}$ and can affect patient's attitude to treatment. Bordin ${ }^{15)}$ conceptualized alliance as consisting of three components: goal agreement, task agreement, and affective bond. Studies have suggested that therapeutic alliance is relevant to therapeutic outcomes, including medication adherence in various psychiatric disorders, such as depression ${ }^{16,17)}$ and bipolar disorder. ${ }^{18)}$ Nonetheless, few studies have explored adherence in schizophrenia. Studies regarding schizophrenia have targeted hospitalized patients ${ }^{12)}$ or those in intensive therapy programs. ${ }^{19,20)}$ In a recent study, McCabe et al. ${ }^{21)}$ investigated the relationship between medication adherence and therapeutic alliance among outpatients with schizophrenia. However, the authors did not consider important clinical factors, such as insight or symptom severity, as covariates. It remains unclear whether the therapeutic alliance from the patient's perspective is associated with medication adherence in an outpatient clinical setting.

Accordingly, this research explored the relationship between medication adherence and therapeutic alliance in patients with schizophrenia attending routine outpatient clinics in Korea. We hypothesized that therapeutic alliance from the patient's perspective would be associated with better medication adherence.

\section{METHODS}

\section{Subjects}

We performed a cross-sectional study of patients aged 18 to 60 years who were diagnosed with schizophrenia according to the Diagnostic and Statistical Manual of Mental Disorders 4th edition, text revision (DSM-IV-TR) and considered to be stable. ${ }^{22)}$ We defined a stable patient as one whose antipsychotic regimen had remained unchanged for the previous 8 weeks and who was able to respond to self-rating psychiatric scales appropriately. Data were collected at two outpatient clinics, namely Sever- ance and Severance Mental Health Hospital. The clinics are located in urban and rural areas of Korea, respectively. Patients were asked to participate in the research by one of the researchers, while patients were waiting to see their clinicians. Patients gave informed, voluntary written consent after the study procedure was fully described to them. This study was approved by the Severance Clinical Review Committee.

\section{Assessments}

To assess medication adherence, patients completed the Medication Adherence Rating Scale (MARS), ${ }^{13)}$ which is a 10-item self-report scale that combines the Medication Adherence Questionnaire ${ }^{23)}$ and Drug Attitude Inventory, ${ }^{24)}$ and which has been validated in Korean patients with schizophrenia. It yields scores ranging from 0 to 10 (perfect adherence).

The therapeutic relationship was assessed using the Scale to Assess the Therapeutic Relationship-Patients version (STAR-P). ${ }^{25)}$ STAR-P is a self-rating measure for patients that comprises 12 questions, each of which is rated on a 5-point Likert scale. Explanatory factor analysis by $\mathrm{Kwon}^{26)}$ with a Korean population revealed the STAR-P has two-factor structures, consisting of 1) "collaborative bond" and 2) "affective bond." Collaborative bond reflects shared understanding of goals and the experience of mutual openness. Affective bond reflects the patient's subjective problems with the relationship, such as the patient's perception that the clinician is impatient, authoritarian, or withholds the truth. The questions addressing the affective bond factor were inverse-scored. A total score was obtained by summing the relevant subscale scores. Higher ratings indicated a better alliance.

Insight was measured using the Scale to Assess Unawareness of Mental Disorder (SUMD). ${ }^{27)}$ SUMD has three items to assess the awareness of having a mental disorder (item 1), the effect of medication (item 2), and the consequences of the mental disorder (item 3). Items are rated from 1 to 5 , with higher ratings indicating lower insight. We used the sum of all items to provide a global insight score.

Ratings of psychopathology were obtained by trained and experienced clinicians when patients agree to participate in the study, using the Brief Psychiatric Rating Scale (BPRS). ${ }^{28)}$ Global functioning was assessed via the Global Assessment of Functioning Scale (GAF). ${ }^{19)}$ Sociodemo- 
graphic characteristics of the participants were also obtained.

\section{Analysis}

Descriptive statistics were obtained for clinical and socio-demographic variables. Associations between the therapeutic alliance and clinical/socio-demographic variables were explored using $t$ tests and bivariate correlations.

We examined the association between therapeutic alliance and medication adherence through regression analysis. We used therapeutic alliance as the independent variable, with covariates potentially associated with adherence of duration of illness, insight, and symptom severity. ${ }^{11)}$ Statistical significance was set at $p<0.05$. All statistical analyses were performed using SPSS version 23 software (IBM Corp., Armonk, NY, USA).

\section{RESULTS}

\section{Participants}

Eighty-one patients participated in the study, of whom $36(44.4 \%)$ were male. The mean age of the participants was 38.02 years (standard deviation [SD], 10.07). Sixty of

Table 1. Clinical characteristics of the study population $(n=81)$

\begin{tabular}{lc}
\hline \multicolumn{1}{c}{ Variable } & Data \\
\hline Symptom severity (BPRS) & $27.31 \pm 9.708(5-50)$ \\
Insight (SUMD) & $7.09 \pm 2.091(3-15)$ \\
Global functioning (GAF) & $52.77 \pm 9.705(50-70)$ \\
Therapeutic alliance (STAR-P) & $45.04 \pm 9.594(26-60)$ \\
Medication adherence (MARS) & $6.81 \pm 2.71(3-15)$ \\
\hline
\end{tabular}

Values are presented as mean \pm standard deviation (range).

BPRS, Brief Psychiatric Rating Scale; SUMD, Scale to Assess Unawareness of Mental Disorder; GAF, Global Assessment of Functioning; STAR-P, Scale to Assess the Therapeutic RelationshipPatients version; MARS, Medication Adherence Rating Scale. the participants were single $(74.1 \%)$. The patients had received treatment for a mean of 11.29 years $(\mathrm{SD}, 7.67)$ and had previously been hospitalized an average of 3.05 times. Table 1 shows the clinical characteristics of the participants.

\section{Association between Therapeutic Alliance and Socio- demographic Characteristics}

There was no difference in therapeutic alliance according to sex and marital status. The results of correlation analysis also showed no significant association between therapeutic alliance and age, illness duration, or admission frequency (data not shown).

\section{Association between Therapeutic Alliance and Other Clinical Variables}

Table 2 shows the results of a correlation analysis between therapeutic alliance and insight, symptom severity, and medication adherence. Therapeutic alliance was not associated with insight but was negatively associated with symptom severity. Of the two factors constituting therapeutic alliance, non-supportive clinical input was associated with medication adherence, but collaboration was not.

\section{Therapeutic Alliance and Adherence}

According to univariate analysis, favorable therapeutic alliance predicted better medication adherence $(B=0.054$, beta $=0.268, \mathrm{t}=2.310, p=0.024)$. Significance was maintained after adjustment for covariates including duration of illness, insight, and symptom severity (Table 3 ).

\section{DISCUSSION}

In the present study, we identified a relationship be-

Table 2. Correlations between therapeutic alliance and clinical variables

\begin{tabular}{|c|c|c|c|}
\hline \multirow{2}{*}{ Variable } & \multicolumn{3}{|c|}{ STAR-P } \\
\hline & Total & Collaborative bond & Affective bond \\
\hline Symptom severity (BPRS) & $-0.236^{*}$ & $-0.288^{*}$ & -0.211 \\
\hline Insight (SUMD) & -0.125 & -0.079 & 0.062 \\
\hline Global functioning (GAF) & 0.060 & 0.014 & 0.099 \\
\hline Medication adherence (MARS) & $0.268^{*}$ & 0.050 & $0.302^{*}$ \\
\hline
\end{tabular}

STAR-P, Scale to Assess the Therapeutic Relationship-Patients version; BPRS, Brief Psychiatric Rating Scale; SUMD, Scale to Assess Unawareness of Mental Disorder; GAF, Global Assessment of Functioning; MARS, Medication Adherence Rating Scale.

Table entries are Pearson correlations coefficients; significant at * $p<0.05$. 
Table 3. Linear regression results for medication adherence by therapeutic alliance

\begin{tabular}{lrrrrc}
\hline & \multicolumn{5}{c}{ Medication adherence (MARS) } \\
\cline { 2 - 5 } & $\mathrm{B}$ & Beta & $\mathrm{t}$ & $p$ & $\mathrm{R}^{2}$ \\
\hline Therapeutic alliance (STAR-P) & 0.060 & 0.277 & 2.526 & 0.014 & 0.318 \\
Duration of illness & 0.097 & 0.392 & 3.693 & 0.000 & \\
Symptom severity (BPRS) & 0.024 & 0.106 & 0.954 & 0.344 & \\
Insight (SUMD) & -0.188 & -0.253 & -2.404 & 0.019 & \\
\hline
\end{tabular}

MARS, Medication Adherence Rating Scale; STAR-P, Scale to Assess the Therapeutic Relationship-Patients version; BPRS, Brief Psychiatric Rating Scale; SUMD, Scale to Assess Unawareness of Mental Disorder.

tween therapeutic alliance and medication adherence in patients with schizophrenia. Patients who reported having a better therapeutic alliance with their clinician showed favorable medication adherence, even after adjustment for factors that might have affected adherence. This is one of the few studies to have investigated the role of therapeutic alliance on medication adherence from the perspective of patients with schizophrenia who were receiving treatment via routine outpatient clinics.

The results of our study are consistent with those of previous studies that investigated the association between therapeutic relationship and medication adherence. ${ }^{12,21)}$ However, the correlation between patient-rated therapeutic alliance and medication adherence was very weak. Moreover, among the two factors that underlie therapeutic alliance, collaboration had no relevance to adherence. This result indicates that active discussions about treatment goals between clinicians and patients had limited impact on adherence. McCabe et al. ${ }^{21)}$ reported that therapeutic alliance from the patient's perspective had a weaker association with adherence than did this alliance from the clinician's perspective. These results suggest that patients with schizophrenia take medication passively via the mental service environment, rather than having their own treatment goals. Among the two components of therapeutic alliance, patients' affective bond showed the stronger association with medication adherence. Lack of affective bond refers to the patient's perception of the clinician as impatient, authoritarian, and liable to withhold the truth. This result is consistent with Day et al.," who showed that patient's coercive experiences during admission were an important determinant of adherence to medication. Nelson et al. ${ }^{29)}$ also found that the patient's perception of the physician's interest in him or her as a person was the single best predictor of medication adherence among discharged pa- tients with schizophrenia. There is a power imbalance between the clinicians and patients in Korea; $75.9 \%$ of patients with schizophrenia are admitted involuntarily. ${ }^{30)}$ This suggests that it is difficult to form positive affective bonds that foster medication adherence with patients with schizophrenia.

The participants showed favorable adherence in this study. Although there is no absolute cutoff value in MARS, the total score of our study was quite high (6.81). When Fialko et al. ${ }^{31)}$ performed large scale validation study with an outpatient clinic, the average score of MARS was 6 . In a survey of patient with homeless schizophrenia in France, the MARS average score was 5.5. ${ }^{32)}$ Since our study was conducted on patients with relatively favorable adherence, further study examining the impact of therapeutic alliance in patients with low adherence is also needed.

In the present study, therapeutic alliance was not associated with insight. This result is inconsistent with other studies; most studies that investigated insight and therapeutic alliance found a significant positive association between these two variables. ${ }^{33)}$ This discrepancy might be because the participants in our study had limited variability in insight scores. Similar results were obtained in other studies that reported low among-patient variance in insight scores. ${ }^{34)}$

There are several difficulties in conducting research on medication adherence in patients with schizophrenia. Medication adherence is not only hard to define, but also difficult to assess. Adherence is a set of behaviors and habits that is dynamic and varies even within individuals, and it cannot be measured dichotomously. Furthermore, since those with poor adherence may also be likely to refuse to participate in a study, selection bias inevitably exists. To solve these difficulties, we used MARS to measure medication adherence continuously, which is a com- 
prehensive tool for evaluating patients' behavior and attitudes. ${ }^{13)}$ Furthermore, since this study was conducted by interviewers who were not involved in the participants' treatment, selection bias should not have been sizable. A further study that considers patients' consent rates may be helpful to understand adherence.

There are some limitations other than the selection bias already mentioned. First, the cross-sectional design limited the ability to draw inferences with respect to the causal direction of associations between adherence and therapeutic alliance, which were considered to be dynamic factors. Second, we did not consider some clinical variables that are known to affect adherence, such as comorbid substance use disorder and treatment regimen. However, since we used insight level as a covariate, we were able to understand more about the impact of therapeutic alliance on adherence. Nevertheless, to our knowledge, this study is one of the few studies to have examined the effect of therapeutic alliance on medication adherence. Moreover, this study reflected the routine outpatient clinical setting and considered confounding factors that could affect adherence.

In conclusion, medication adherence among patients with schizophrenia patients who were treated in a routine outpatient clinic was influenced by therapeutic alliance. These findings suggest that it is important to establish a genuine and humanistic relationship between clinicians and patients to improve medication adherence.

\section{Acknowledgments}

The authors would like to thank all of the staff at Severance Hospital for their assistance with this research.

\section{REFERENCES}

1. Naber D, Karow A. Good tolerability equals good results: the patient's perspective. Eur Neuropsychopharmacol 2001;11 Supp/ 4:S391-S396.

2. Park SC, Choi MY, Choi J, Park E, Tchoe HJ, Suh JK, et al. Comparative efficacy and safety of long-acting injectable and oral second-generation antipsychotics for the treatment of schizophrenia: a systematic review and meta-analysis. Clin Psychopharmacol Neurosci 2018;16:361-375.

3. Verdoux H, Lengronne J, Liraud F, Gonzales B, Assens F, Abalan F, et al. Medication adherence in psychosis: predictors and impact on outcome. A 2-year follow-up of first-admitted subjects. Acta Psychiatr Scand 2000;102:203-210.

4. Babiker IE. Noncompliance in schizophrenia. Psychiatr Dev
1986;4:329-337.

5. Blackwell B. Drug therapy: patient compliance. NEng/JMed 1973;289:249-252.

6. Ascher-Svanum H, Zhu B, Faries DE, Salkever D, Slade EP, Peng $\mathrm{X}$, et al. The cost of relapse and the predictors of relapse in the treatment of schizophrenia. BMC Psychiatry 2010;10:2.

7. Day JC, Bentall RP, Roberts C, Randall F, Rogers A, Cattell D, et al. Attitudes toward antipsychotic medication: the impact of clinical variables and relationships with health professionals. Arch Gen Psychiatry 2005;62:717-724.

8. Kao YC, Liu YP. Compliance and schizophrenia: the predictive potential of insight into illness, symptoms, and side effects. Compr Psychiatry 2010;51:557-565.

9. Murru A, Pacchiarotti I, Nivoli AM, Bonnin CM, Patrizi B, Amann B, et al. Rates and clinical correlates of treatment non-adherence in schizoaffective bipolar patients. Acta Psychiatr Scand 2012;125:412-418.

10. Sendt KV, Tracy DK, Bhattacharyya S. A systematic review of factors influencing adherence to antipsychotic medication in schizophrenia-spectrum disorders. Psychiatry Res 2015;225: 14-30.

11. Lacro JP, Dunn LB, Dolder CR, Leckband SG, Jeste DV. Prevalence of and risk factors for medication nonadherence in patients with schizophrenia: a comprehensive review of recent literature. J Clin Psychiatry 2002;63:892-909.

12. Misdrahi D, Petit M, Blanc O, Bayle F, Llorca PM. The influence of therapeutic alliance and insight on medication adherence in schizophrenia. Nord J Psychiatry 2012;66:49-54.

13. Thompson K, Kulkarni J, Sergejew AA. Reliability and validity of a new Medication Adherence Rating Scale (MARS) for the psychoses. Schizophr Res 2000;42:241-247.

14. Svensson B, Hansson L. Relationships among patient and therapist ratings of therapeutic alliance and patient assessments of therapeutic process: a study of cognitive therapy with long-term mentally ill patients. I Nerv Ment Dis 1999;187: 579-585.

15. Bordin ES. The generalizability of the psychoanalytic concept of the working alliance. Psychother Theory Res Pract 1979;16:252-260.

16. Krupnick JL, Sotsky SM, Simmens S, Moyer J, Elkin I, Watkins J, et al. The role of the therapeutic alliance in psychotherapy and pharmacotherapy outcome: findings in the National Institute of Mental Health Treatment of Depression Collaborative Research Program. J Consult Clin Psychol 1996;64:532-539.

17. Weiss M, Gaston L, Propst A, Wisebord S, Zicherman V. The role of the alliance in the pharmacologic treatment of depression. J Clin Psychiatry 1997; 58:196-204.

18. Gaudiano BA, Miller IW. Patients' expectancies, the alliance in pharmacotherapy, and treatment outcomes in bipolar disorder. J Consult Clin Psychol 2006; 74:671-676.

19. Neale MS, Rosenheck RA. Therapeutic alliance and outcome in a VA intensive case management program. Psychiatr Serv 1995;46:719-721. 
20. Frank AF, Gunderson JG. The role of the therapeutic alliance in the treatment of schizophrenia. Relationship to course and outcome. Arch Gen Psychiatry 1990;47:228-236.

21. McCabe R, Bullenkamp J, Hansson L, Lauber C, Martinez-Leal $\mathrm{R}$, Rössler W, et al. The therapeutic relationship and adherence to antipsychotic medication in schizophrenia. PLoS One 2012; 7:e36080.

22. American Psychiatric Association. Diagnostic and statistical manual of mental disorders: 4th ed, text revision (DSM-IV-TR). Washington, D.C.:American Psychiatric Association;2000.

23. Morisky DE, Green LW, Levine DM. Concurrent and predictive validity of a self-reported measure of medication adherence. Med Care 1986;24:67-74.

24. Hogan TP, Awad AG, Eastwood R. A self-report scale predictive of drug compliance in schizophrenics: reliability and discriminative validity. Psychol Med 1983;13:177-183.

25. McGuire-Snieckus R, McCabe R, Catty J, Hansson L, Priebe S. A new scale to assess the therapeutic relationship in community mental health care: STAR. Psychol Med 2007;37:85-95.

26. Kwon JY. Validation study on the utilization of the Korean version of the STAR scale. Ment Health Soc Work 2010;35: 81-110.

27. Amador XF, Strauss DH, Yale SA, Flaum MM, Endicott J, Gorman JM. Assessment of insight in psychosis. AmJ Psychiatry 1993;150:873-879.
28. Overall JE, Gorham DR. The brief psychiatric rating scales. Psychol Rep 1962;10:799-812.

29. Nelson AA Jr, Gold BH, Huchinson RA, Benezra E. Drug default among schizophrenic patients. Am J Hosp Pharm 1975; 32:1237-1242.

30. Seoul Mental Health Statistics. Involuntary admission in high income country [Internet]. Seoul: Seoul Mental Health Statistics; 2015 [cited at 2018 May 30]. Available from: https://www.seoulmentalhealth.kr/about/trend2.jsp.

31. Fialko L, Garety PA, Kuipers E, Dunn G, Bebbington PE, Fowler $\mathrm{D}$, et al. A large-scale validation study of the Medication Adherence Rating Scale (MARS). Schizophr Res 2008;100: 53-59.

32. Zemmour $\mathrm{K}$, Tinland A, Boucekine $\mathrm{M}$, Girard V, Loubière $\mathrm{S}$, Resseguier $\mathrm{N}$, et al. Validation of the Medication Adherence Rating Scale in homeless patients with schizophrenia: results from the French housing first experience. Sci Rep 2016;6: 31598.

33. Shattock L, Berry K, Degnan A, Edge D. Therapeutic alliance in psychological therapy for people with schizophrenia and related psychoses: a systematic review. Clin Psychol Psychother 2018;25:e60-e85.

34. Jung E, Wiesjahn M, Rief W, Lincoln TM. Perceived therapist genuineness predicts therapeutic alliance in cognitive behavioural therapy for psychosis. Br JClin Psychol 2015;54:34-48. 